\title{
La Medicina tradicional peruana en la obra de Ricardo Palma
}

Por César Coloma Porcari 
Presidente del Instituto Latinoamericano de Cultura y Desarrollo y Miembro de Número del Instituto Ricardo Palma. 
Las Tradiciones de don Ricardo Palma ofrecen la más amplia información sobre el pasado limeño, incluyendo la medicina tradicional peruana, referente a la cual brinda escasa pero, a la vez, muy valiosa información. En este trabajo, presentamos veintiún plantas medicinales mencionadas por Palma -que se aplicaban para curaciones específicas- que él también describe.

Debemos recordar que en su tradición “iA nadar, peces!", se ocupa de una típica farmacia limeña cuando nos cuenta que un mozalbete le ofreció a un libertino fraile poner una botica, si el sacerdote le entregaba todo el dinero necesario para ello. Le dijo a su amigo clérigo que se necesitaba comprar "Un almirez, un morterito de piedra, una retorta, un alambique, un tarro de sanguijuelas, unas cuantas onzas de goma, linaza, achicoria y raíz de altea, unos frascos vistosos, vacíos los más y pocos con drogas, y pare usted de contar" (Tradiciones Peruanas completas, Madrid, Aguilar S. A. de Ediciones, 1968: 843, 844).

Las diversas plantas medicinales empleadas en el Perú, que Palma menciona (y que hemos ordenado alfabéticamente, con el respectivo nombre científico, para facilitar la consulta de los interesados), son las siguientes:

Achicoria (Chicorium intibus L.).-

En "El primer toro" (Palma 1968: 653), cuenta que en los inicios de la Independencia se intentó suprimir el gasto en 
"cerveza y butifarras" para agasajar a las más altas autoridades que acudían a las corridas en la plaza de toros de Acho, mediante un "decretito de agua de malvas, achicoria y raíz de altea", porque la mencionada norma "no otorgaba concesión ni implicaba negativa rotunda". Y en "iA nadar, peces!" (Palma 1968: 843, 844), se refiere a un poco virtuoso sacerdote que fue engañado por un amigo, libertino como él, al establecer un negocio de botica, con su propio dinero. Compró "achicoria" y otras hierbas e implementos para el negocio.

\section{Altea (Altcea officinalis L.).-}

Nuestro gran escritor, en "El primer toro" (Palma 1968: 653), se refiere al deseo de suprimir el pago para agasajar a las autoridades que acudían a presenciar las corridas de toros en la plaza de Acho, en los primeros años de la Independencia. Anteriormente se les había ofrecido "cerveza y butifarras". Se intentó eliminar el gasto a través de un "decretito de agua de malvas, achicoria y raíz de altea", llamado así por Palma porque ese dispositivo "no otorgaba concesión ni implicaba negativa rotunda". Asimismo, en "iA nadar, peces!" (Palma 1968: 843, 844), afirma que a un poco casto sacerdote lo engañó un mozo jaranista, amigo suyo, al convencerlo para poner juntos una botica (con el dinero del fraile). Compró éste "raíz de altea", unos frascos vistosos, vacíos los más y pocos con drogas, y pare usted de contar". Don Antonio Raimondi afirma que la altea es "indígena de Europa, y conocida con el nombre de Malvavisco; la raíz de esta planta es la que tiene la mayor proporción de principios mucilaginosos, y contiene, además, un principio cristalizable, análogo a la Esparagina" (Raimondi 1857: 265). 
Borraja (Borago officinalis L.).-

Afirma el tradicionista, en "El latín de una limeña" (Palma 1968: 628), al referirse a las antiguas creencias de los limeños, que "el Credo se reputaba como remedio cálido, y era mejor sudorífico que el agua de borrajas". El sabio don Antonio Raimondi registra que "Esta planta es cultivada por todas partes, tanto como planta de adorno por sus corolas de un hermoso color azul, cuanto por sus propiedades diuréticas y sudoríficas, debido a una cierta cantidad de nitro que contiene" (Raimondi 1857: 149).

\section{Cascarilla o Quina (Cinchona officinalis L.).-}

En su tradición "Los polvos de la condesa" (Palma 1968: $355-$ 357), nos cuenta que la esposa del virrey conde de Chinchón, en 1639, se encontraba en peligro de muerte, por encontrarse gravemente enferma. Estando el virrey en la habitación de su señora, escuchó una voz que decía "Se salvará la condesa, excelentísimo señor". Entonces "El virrey se volvió, sorprendido. Era un sacerdote, hijo de Ignacio de Loyola, el que había pronunciado tan consoladoras palabras". El fraile agregó: "Quiero ver a la virreina; tenga vuecencia fe, y Dios hará el resto". Palma nos habla del descubrimiento de los poderes curativos de la quinina, cuando "Atacado de fiebres un indio de Loja, llamado Pedro de Leiva, bebió, para calmar los ardores de la sed, del agua de un remanso, en cuyas orillas crecían algunos árboles de quina. Salvado así, hizo la experiencia de dar de beber a otros enfermos del mismo mal, cántaros de agua, en la que depositaba raíces de cascarilla. Con su descubrimiento vino a Lima y lo comunicó a un jesuita, el que, realizando la feliz curación de la virreina, prestó a la Humanidad mayor servicio que el fraile que inventó la pólvora”. El tradicionista 
agrega que "Los jesuitas guardaron por algunos años el secreto, y a ellos acudía todo el que era atacado de tercianas. Por eso, durante mucho tiempo, los polvos de la corteza de quina se conocieron con el nombre de polvos de los jesuitas". Menciona Palma, inclusive, que un médico inglés curó con la quinina, a prominentes personajes franceses, y que Carolus Linnæus, en homenaje a la condesa de Chinchón, curada por la cascarilla, la denominó científicamente con ese nombre: Cinchona (y no "Chinchona" como el vulgo cree).

Don Antonio Raimondi afirma que "El género Chincona comprende muchas especies útiles a la Medicina, suministrando las preciosas cortezas que se conocen en el comercio con el nombre de Cascarilla, de la que se extrae el más activo y seguro febrífugo que posee la Terapéutica, esto es, la Quinina". Agrega que "Para beneficiar la Cascarilla se usa cortar el árbol casi a raíz de la tierra, quitarle su epidermis, dividir su corteza en muchas tiritas, que se despegan por medio de un cuchillo, y en seguida se hacen secar al sol; la corteza de las pequeñas ramas, siendo delgada, se enrosca sobre sí misma, y constituye la Cascarilla en canuto; la de las ramas más grandes y la del tronco, se somete a una presión, de modo que quede en pedazos llanos. En fin, para expedirla a Europa, se envuelve la corteza en cueros frescos, los que, secándose, se contraen y adquiere una gran solidez y constituyen los fardos que se conocen con el nombre de zurrones" (Raimondi 1857: 194, 196).

Chamico (Datura stramonium L).-

El tradicionista, en "Un obispo de Ayacucho" (Palma 1968: 698), cuenta que "Al obispo fray Antonio Conderino, a poco de haberse hecho cargo de la diócesis, en 1645, le dieron chamico, y murió en el convento agustino de Lima”. Además, en 
"Supersticiones de los peruanos" (Palma 1968: 1433), registra que "tener bajo una olla un sapo ciego, convertir en alfiletero un muñeco de trapo, y otras brujerías con las que hacen daño o hechizan a una persona, es superstición muy generalizada. No lo es menos la del chamico, hierba que entontece o vuelve loco a un hombre". En "Neologismos y americanismos" afirma que "chamico" es voz quechua y que es una "Yerba que administran los indios para entontecer a una persona. También la usan como afrodisiaco" (Palma 1896: 27). Lo mismo señala en "Papeletas lexicográficas" (Palma 1903: 65).

El sabio don Antonio Raimondi afirma que "La Datura stramonium, que se conoce con el nombre vulgar de Chamico, es una planta venenosa, que se distingue de las demás especies, por su corola blanca, con la parte interna del tubo de color morado, y por sus frutos espinosos. La raíz de esta planta, rascada y fumada con el tabaco, ha sido preconizada contra el asma" (Raimondi 1857: 158).

Coca (Erythroxylon coca Lamarck).-

El gran escritor, en "Supersticiones de los peruanos" (Palma 1968: 1431), dice que un brujo, para curar, emplea "cuatro onzas de coca, maíz, cebada, trigo, habas y quinua" y otros elementos. "Otros brujos toman un puñado de coca", la soplan "y diagnostican según la dirección que han tomado las hojitas". Don Antonio Raimondi, al referirse a la coca, afirma que "algunos han supuesto que es [un] narcótico, pero esta hipótesis está destruida desde que se sabe, por el contrario, que produce insomnio; otros piensan que está cargada de principios nutritivos y que es una substancia alimenticia. Si bien es verdad que la Coca contiene principios azoados y que a éstos se debe la propiedad de mantener las fuerzas por algunas 
horas, si se considera la cantidad tan pequeña que consumen en el día, se vendrá en cuenta de que no puede bastar para la alimentación. La opinión más generalmente admitida es que tiene propiedades excitantes, pero con la especialidad de no obrar como el Café, el Té \&c., con una acción localizada, sino, como lo ha hecho notar el doctor Weddel, de un modo difuso y lento, sobre todo el sistema nervioso" (Raimondi 1857: 257).

Congona (Peperomia inoxqualifolia Ruiz \& Pavon; Peperomia congona Ruiz \& Pavon).

El autor, en "De esta capa nadie escapa” (Palma 1968: 855), se refiere a la hija del virrey Abascal, doña Ramona, que "era tan linda como mimada y melindrosa". Cuenta que por cualquier motivo "teníamos a Ramoncica con soponcio y a su buen padre, el excelentísimo señor virrey de estos reinos del Perú y Chile, gritando como loco y corriendo tras la hoja de congona, el frasquito de alcalinas o el agua de melisa”.

Culén.- (Psoralea glandulosa L.).-

Nuestro gran tradicionista, en "Los panecitos de San Nicolás" (Palma 1968: 293), cuenta de una enferma grave a la que le administraron los milagrosos "panecitos de San Nicolás", que no surtieron ningún efecto en ella. "La enferma siguió retorciéndose, sin que las lavativas ni el agua de culén o de hierbaluisa [vide] la aliviaran en lo menor". Los familiares amenazaron, entonces, a San Nicolás de Tolentino, que le rezarían a fray Francisco Solano, que se encontraba en proceso de canonización, si no curaba a la enferma. "La suerte favoreció a San Nicolás. Una nueva lavativa, en la que se desmenuzó un panecito, bastó para desatracar cañerías". Don Antonio 
Raimondi afirma que "la Psoralea glandulosa, indígena del Perú, donde se conoce con el nombre vulgar de Culén, y se usa en infusión teiforme en las indigestiones, contra los cólicos y las lombrices intestinales" (Raimondi 1857: 218).

Floripondio (Datura arborea L.).-

Cuenta en "La monja de la llave" (Palma 1968: 206), que doña Violante, una señorita celosamente cuidada por doña Circuncisión, se libraba de ésta cada vez que quería divertirse con un galán, para lo cual echaba en el chocolate de la anciana "algunas gotas de extracto de floripondios, que producían en la beata un sueño que distaba no mucho del eterno". El sabio do Antonio Raimondi afirma que "La tribu de las Datureas comprende algunas plantas venenosas, de flores grandes, cuyos frutos son a veces espinosos, y contienen semillas dotadas también de principios narcóticoacres muy activos, debido a un alcaloide cristalizable llamado Daturina, que tiene la singular propiedad de fijar y dilatar la pupila del ojo" (Raimondi 1857: 158).

Goma arábiga.- (Acacia vera Bauhin; A. gummifera Willdenow; A. arabica Willdenow).-

En "La emplazada" (Palma 1968: 473), cuenta de una esclava, Gertrudis, que fue trasladada a la hacienda de su ama. Establecida allí, “despertó en el capellán y el médico todo el apetito que inspira una golosina". Además, "Su reverencia frailuna dio en padecer de distracciones cuando abría su libro de horas; y el médico-boticario se preocupó con la mocita a extremo tal que, en cierta ocasión, administró a uno de sus enfermos jalapa [vide] en vez de goma arábiga, y en un tumbo de dado estuvo que lo despachase sin postillón al país de las calaveras". 
Y en “iA nadar, peces!" (Palma 1968: 843, 844), relata que un joven aventurero convenció a un poco casto sacerdote para poner juntos una botica, si el fraile aportaba todo el capital. Para ello era necesario adquirir "unas cuantas onzas de goma" y otros productos. El sabio don Antonio Raimondi registra: "Las Acacia vera, gummifera y arabica, que suministran la goma tan usada como pectoral y conocida en el comercio con el nombre de Groma arábiga. Estas especies de acacias son pequeños árboles de hojas bipinnadas, con dos espinas estipulares y sus legumbres con bordes sinuosos" (Raimondi 1857: 220).

Hierba santa (Cestrum hediondum Ruiz \& Pavon; Cestrum auriculatum Ruiz \& Pavon).-

Afirma en "El abad de Lunahuaná" (Palma 1968: 199), que el Papa Gregorio XIII, en 1581, llevaba dos meses de estar "postrado en su lecho", atacado de "caries maxilar", que era mortal en ese entonces. Dio la casualidad que se encontraba en Roma fray Miguel de Carmona, Definidor del convento agustino de Lima. Este sacerdote solicitó acercarse al Papa para que "intentara su curación empleando hierbas que había traído del Perú, y cuya eficacia entre los naturales de América para dolencias tales le constaba". El Papa aceptó "Y ello fue que sin más que enjuagatorios de hierba santa con leche, cataplasmas de llantén [vide] con vinagrillo y parches de tabaco bracamoro [vide] en las sienes, a los tres días estuvo Su Santidad Gregorio XIII como nuevo, y tanto, que hasta la hora de su muerte, que acaeció años más tarde, no volvió a dolerle muela ni diente". Afirma don Antonio Raimondi que esta planta "nace espontánea, en mucha abundancia, en las cercanías de Lima, a donde se conoce con el nombre de Yerba Santa o de Yerba hedionda, y se emplea en infusión, como refrigerante y emoliente, o también, para lavar las úlceras" (Raimondi 1857: 162). 
Hierbaluisa (Cymbopogon citratus De-Candolle).-

Dice en "Los panecitos de San Nicolás" (Palma 1968: 293) que doña Ana de Urdanivia "tomóse un atracón que le produjo un cólico miserere". Pero "La enferma siguió retorciéndose, sin que las lavativas ni el agua de culén [vide] o de hierbaluisa la aliviaran en lo menor". La familia le advirtió a San Nicolás de Tolentino que se encomendaría entonces a fray Francisco Solano, contribuyendo con dinero para su canonización, si no curaba a la enferma. El santo Tolentino debe haberse quedado preocupado porque "Una nueva lavativa, en la que se desmenuzó un panecito, bastó para desatracar cañerías". El sabio don Antonio Raimondi no menciona la hierbaluisa en su tratado de Botánica (Raimondi 1857).

\section{Huamanripa (Cryptochaete andicola Raimondi).-}

Nuestro tradicionista, en "Papeletas lexicográficas", afirma que "huamanripa" es voz quechua, y es una "Planta americana, muy usada para combatir las afecciones bronquiales" (Palma 1903: 140). Don Antonio Raimondi designó esta planta con el nombre de Cryptochaete andicola, y afirma que "es una compuesta que crece en los lugares muy frígidos de la cordillera del Perú; es conocida por los indígenas con el nombre de Huamanripa, y forma uno de los principales medicamentos contra las hemorragias y las enfermedades del pulmón" (Raimondi 1857: 187).

Jalapa (Exagonium purga Bentham; Ipomoea jalapa Nuttall)

En "Entre jesuitas, agustinos y dominicos" (Palma 1968: 215), afirma que "En aquellos tiempos las excomuniones andaban bobas y producían menos efecto que los polvos de Jalapa, 
purgativo a la moda". En "La emplazada" (Palma 1968: 473), se refiere a Gertrudis, una joven esclava africana que al llegar a la hacienda de su ama, "despertó en el capellán y el médico todo el apetito que inspira una golosina". Asimismo, "Su reverencia frailuna dio en padecer de distracciones cuando abría su libro de horas; y el médico-boticario se preocupó con la mocita a extremo tal que, en cierta ocasión, administró a uno de sus enfermos jalapa en vez de goma arábiga [vide], y en un tumbo de dado estuvo que lo despachase sin postillón al país de las calaveras". Afirma don Antonio Raimondi que esta planta, indígena de México, "es la que suministra la verdadera jalapa usada en Medicina; tiene por caracteres específicos una raíz tuberosa, redonda, de color negruzco en el exterior, blanquizco en el interior, y llena de un jugo lechoso de naturaleza resinosa, dotado de propiedades purgantes muy activas" (Raimondi 1857: 147).

Linaza (Linum usitatissimum L.).-

El tradicionista, en “iA nadar, peces!” (Palma 1968: 843, 844), se refiere a un mozo estafador que engañó a un sacerdote no muy casto, al animarlo a poner, con él, una botica, como negocio. El cura aportó todo el capital, adquiriendo, entre otros productos "linaza", algunas hierbas y envases. El sabio don Antonio Raimondi afirma que "El Linum usitatissimum" es una "pequeña planta de tallo simple, hijas pequeñas y flores azules, cultivada en los lugares inundados de Europa desde tiempo inmemorial, porque las fibras muy tenaces de su tallo suministran la materia textil más usada, esto es, el lino, que sirve, como todos saben, en la fabricación de las telas de lujo. Las semillas de la misma especie, conocidas en Lima con el nombre de Linaza, son mucilaginosas, y reducidas en harina, son empleadas en cataplasmas; contienen, además, una cantidad 
bastante notable de aceite, muy usado en la pintura y en las artes" (Raimondi 1857: 255).

\section{Llantén (Plantago major L.).-}

Nos cuenta en "El abad de Lunahuaná” (Palma 1968: 199), que en 1581 el Papa Gregorio XIII llevaba dos meses de estar "postrado en su lecho", por causa de una "caries maxilar", mortal en ese entonces. Se encontraba en Roma el agustino fray Miguel de Carmona, del convento de Lima. Solicitó este que se "intentara su curación empleando hierbas que había traído del Perú, y cuya eficacia entre los naturales de América para dolencias tales le constaba". Aceptó el Papa "Y ello fue que sin más enjuagatorios de hierba santa [vide] con leche, cataplasmas de llantén con vinagrillo y parches de tabaco bracamoro [vide] en las sienes, a los tres días estuvo Su Santidad Gregorio XIII como nuevo, y tanto, que hasta la hora de su muerte, que acaeció años más tarde, no volvió a dolerle muela ni diente". Afirma don Antonio Raimondi que "Muchas especies de llantén tienen propiedades ligeramente astringentes, que las hacían usar, en otro tiempo, en la medicina" (Raimondi 1857: 114).

\section{Malva (Lavatera arborea L.).-}

Nuestro tradicionista, en "El primer toro" (Palma 1968: 653), afirma que se trató de eliminar el agasajo que con "cerveza y butifarras" se ofrecía a las autoridades que asistían a las corridas de toros en la plaza de Acho, a través de un "decretito de agua de malvas, achicoria y raíz de altea”. Palma lo calificó así debido a que la norma "no otorgaba concesión ni implicaba negativa rotunda". En "Una astucia de Abascal" (Palma 1968: 845), dice que "las protestas del débil contra el fuerte son agua de malvas", 
y en "El poeta de las adivinanzas", un personaje cuenta que tomó "Agua de malvas en lugar de chocolate" (Palma 1968: 721722). Registra el sabio don Antonio Raimondi que "La mayor parte de las Malváceas contienen principios mucilaginosos, que las hacen usar como emolientes". En cuanto a la Lavatera arborea L., afirma que "es una malvácea de grande talla, indígena de Europa y cultivada en todas las huertas de Lima, donde se conoce con el nombre de Malva, y se emplean sus flores, hojas y raíces, como emoliente" (Raimondi 1857: 265-266).

\section{Melisa (Melissa officinalis L.).-}

En "La emplazada" (Palma 1968: 471), se refiere al "ocio y aislamiento" al vivir en una hacienda y que para calmar "los nervios siempre impresionables en las hijas de Eva", existía la "confianza que para calmarlos se tiene en el agua de melisa". Además, en "Una astucia de Abascal" (Palma 1968: 846), se refiere a doña Ramona, la hija del virrey Abascal, señalando que era muy "melindrosa". Un día le dio "pataleta" al ver una pequeña araña sobre el tapiz de una pared del salón. Su padre el virrey mandó que le dieran "agua de melisa". Y en "De esta capa nadie escapa" (Palma 1968: 855), cuenta que doña Ramona Abascal, la hija del virrey, fue la primera mujer "que trajo a Lima la moda de los ataques de nervios y demás arrechuchos femeniles", y a ella en cualquier momento le daba un "soponcio", y para calmarla, le daban "agua de melisa". Don Antonio Raimondi, al referirse a la Melissa officinalis L., señala que pertenece a la familia de las Labiate (Labiadas), indicando que sus hojas "están provistas de un gran número de glándulas llenas de un aceite esencial al que deben su olor aromático", y se emplean "para la preparación de aguas destiladas usadas en la medicina como estimulantes". Agrega que el nombre vulgar de la Melissa officinalis L. es "toronjil". 
Melón (Cucumis melo L.).-

Afirma el ilustre tradicionista, en "El latín de una limeña" (Palma 1968: 628), que no hay "cosa más fresca y desirritante que una horchata de pepitas de melón".

\section{Tabaco bracamoro (Nicotiana tabacum L.).-}

Nos cuenta en "El abad de Lunahuaná" (Palma 1968: 199), que el Papa Gregorio XIII, en 1581, llevaba dos meses de estar "postrado en su lecho", por la en ese entonces mortal "caries maxilar". Casualmente se encontraba en Roma fray Miguel de Carmona, agustino de Lima, quien ofreció curarlo "empleando hierbas que había traído del Perú, y cuya eficacia entre los naturales de América para dolencias tales le constaba". El Papa aceptó "Y ello fue que sin más enjuagatorios de hierba santa [vide] con leche, cataplasmas de llantén [vide] con vinagrillo y parches de tabaco bracamoro en las sienes, a los tres días estuvo Su Santidad Gregorio XIII como nuevo, y tanto, que hasta la hora de su muerte, que acaeció años más tarde, no volvió a dolerle muela ni diente". Registra don Antonio Raimondi que "Las hojas del tabaco son acres, eméticas y drásticas; cuando se usan en cierta cantidad, son estupefacientes, causan delirio, convulsiones y la muerte" (Raimondi 1857: 157).

Valeriana (Valeriana officinalis L.).-

El tradicionista don Ricardo Palma, en "Supersticiones de los peruanos", refiere que "sobre los gatos influye el olor de la valeriana" (Palma 1968: 1431). El sabio don Antonio Raimondi afirma que "La Valeriana officinalis o Valeriana común [...] es una planta indígena de Europa y también de los cerros que 
rodean Lima; de vara a vara y media de alto, de hojas opuestas y pinnadas. Sus flores son pequeñas y numerosas, de un color blanco purpurino. Sus raíces tienen un olor nauseabundo y constituyen uno de los mejores antiespasmódicos" (Raimondi 1857: 181).

\section{Información adicional.-}

\section{Raíces diversas.-}

En "La trenza de sus cabellos" (Palma 1968: 553), afirma que "La antigua limeña no usaba elíxires odontálgicos [sic] ni polvos para los dientes; y sin embargo, era notable la regularidad y limpieza de éstos". "¿Saben ustedes a quién debía la limeña la blancura de sus dientes? Al raicero. Como el jazminero, era éste otro industrioso ambulante que vendía ciertas raíces blandas y jugosas, que las jóvenes se entretenían en morder, restregándolas sobre los dientes". Lamentablemente el tradicionista no indica a qué raíces se refería, y por ello nos ha privado de un valioso remedio natural para la dentadura.

\section{Vinagre de los siete ladrones.-}

Se refiere a este líquido misterioso en "Una astucia de Abascal" (Palma 1968: 846), al recordar a doña Ramona, la hija del virrey don Fernando de Abascal, indicando que era muy nerviosa. En una oportunidad le dio un ataque de nervios al ver una pequeña araña sobre el tapiz de una pared del salón. El virrey mandó que le dieran el frasquito con el "vinagre de los siete ladrones, que es santo remedio contra los nervios". 
Era éste un antiguo preparado medicinal europeo y empleado en la magia y brujería, el Acetum prophylacticum seu latronum. Se conoce en inglés como "vinegar of the seven thieves", en italiano "aceto dei sette ladri", en francés "vinaigre des sept voleurs", y en castellano, "vinagre de los siete ladrones" o "vinagre de los cuatro ladrones". Está compuesto, generalmente, de ajenjo (Artemisia absinthium L.), ajo (Allium sativum L.), alcanfor (Camphora officinarum Nees), cálamo aromático (Acorus calamus L.), canela (Cinnamomum zeylanicum Breyn), clavo de olor (Caryophyllus aromaticus L.), espliego (Lavandula vera DeCandolle), menta (Mentha piperita L.), nuez moscada (Myristica moschata Thunberg), romero (Rosmarinus officinalis L.), ruda (Ruta graveolens L.), salvia (Salvia officinalis L.), y vinagre. El Dr. François-Laurent-Marie Dorvault afirma que "Esta composición se usa como preservativo en las infecciones contagiosas. Se frotan con ella las manos y la cara; se quema o riega en las habitaciones, y también se pone en frasquitos, para hacerla aspirar, en el síncope y la asfixia" (Dorvault 1859: 835).

\section{Conclusiones.-}

Las referencias que hace don Ricardo Palma a la curación de diversos males, por medio del empleo de diversas plantas medicinales, son una valiosa fuente de información verificada por los datos que proporciona don Antonio Raimondi. Por dicha razón, tanto las plantas medicinales como el uso que tenían antaño, deberían ser estudiados científicamente a fin de determinar la veracidad de sus propiedades curativas.

Se debe tener presente que la única manera de comprobar la eficacia de los tratamientos mencionados en esta estudio, es realizando un trabajo de investigación a cargo de competentes especialistas en medicina, química, etc. Su resultado puede ser 
de gran utilidad ya que es muy probable que estas plantas sí tengan virtudes curativas, y que su uso en la actualidad podría ser muy apreciado por quienes prefieren las dietas saludables y el consumo de productos naturales.

\section{Bibliografía.-}

COLOMA PORCARI, César: "La Flora del Perú en las Tradiciones de Ricardo Palma", en "Aula Palma", vol. X, Lima, Instituto Ricardo Palma, Universidad Ricardo Palma, 2011, pp. 59-90.

DORVAULT, François-Laurent-Marie: La Botica o repertorio general de Farmacia práctica, Madrid, Librería Extranjera y Nacional, Científica y Literaria, 1859.

PALMA, Ricardo: Tradiciones Peruanas completas, Madrid, Aguilar S. A. de Ediciones, 1968.

Neologismos y americanismos, Lima, Imprenta y Librería de Carlos Prince, 1896.

Papeletas Lexicográficas, Lima, Imprenta La Industria, 1903.

RAIMONDI, Antonio: Elementos de Botánica aplicada a la Medicina y a la Industria, en los cuales se trata especialmente de las plantas del Perú, "Segunda parte. Taxonomía, Fitografía y Geografía Botánica”, Lima, Tipografía calle del Compás № 202, 1857.

SOUKUP, Jaroslav: Vocabulario de los nombres vulgares de la Flora peruana, Lima, Escuela Tipográfica Salesiana, 1971. 